adiposity in the neonate $[S S+T R p=0.003, p=0.008$ respectively; Sum of skinfolds $\mathrm{p}=0.011, \mathrm{p}=0.002$ respectively] [Table 1]. Maternal early and late pregnancy $\mathrm{TNF} \alpha$ was also associated with 6-month-old central adiposity [SS/TR ratio $\mathrm{p}=$ $0.002, \mathrm{p}=0.030$, respectively]. Fetal TNF $\alpha$ did not exert a significant influence on neonatal anthropometry but was associated with infant triceps skinfold at 6 months.

While fetal IL-6 was associated with birth length and waist: height ratio, maternal IL-6 was not significantly associated with adiposity.

On multiple linear regression analysis, TNF $\alpha$ contributed significantly to the majority of the final models for both neonatal and infant anthropometry, however, the models themselves were not significant. Any associations between maternal or fetal IL-6 with neonatal and infant anthropometry were no longer significant in the multiple regression models.

Conclusion Maternal TNF $\alpha$ significantly correlates with greater offspring adiposity at birth and 6 months and therefore may act as a potential antenatal indicator of a predisposition towards early childhood obesity.

\section{G314(P) CAN MULTIDISCIPLINARY SIMULATION IN A PAEDIATRIC DEPARTMENT IMPROVE CLINICAL GOVERNANCE?}

${ }^{1} \mathrm{D}$ Birahinduka, ${ }^{1} \mathrm{H}$ Salam, ${ }^{1} \mathrm{C}$ Petropoulos, ${ }^{2} \mathrm{~S}$ Trippick, ${ }^{1} \mathrm{~K}$ Baillie. ${ }^{1}$ Paediatrics, University College Hospital London, London, UK; ${ }^{2}$ Emergency Medicine, University College Hospital London, London, UK

10.1136/archdischild-2015-308599.291

Background Simulation is increasingly becoming an integral part in paediatric teaching. It offers an excellent opportunity to practice medical emergencies in a safe environment and allows addressing human factors that are pivotal in safely managing the critically ill child. However, multidisciplinary simulation in our paediatric unit has also facilitated discussion about clinical governance issues within the wider institution.

Aims Does multidisciplinary simulation program in paediatrics address clinical governance issues?

Methods Over a four month period ad hoc in house multidisciplinary simulation sessions involving medical and nursing staff of all levels from the paediatric, anaesthetic, radiology and emergency medicine department were conducted. The simulation sessions were held on paediatric wards and in the emergency department. Faculty members were resuscitation officers, paediatricians, anaesthetists and nurses with experience in conducting simulation. The sessions lasted 20-25 min; followed by debrief for another $20 \mathrm{~min}$. Immediate feedback on the simulation sessions and learning points was obtained. The quality, the usefulness and the conduct of the simulation were evaluated in a feedback form.

Results In total, thirteen simulation sessions were held over a four month period and there were an average of 11 attendees per session. During immediate discussions areas of concern were identified. These included clinicians' performance, human factors and clinical governance issues. Governance risks included inadequate resuscitation equipment and emergency protocol folder, access to emergency lifts, and the quality of emergency activation system.

Conclusions Multidisciplinary simulation sessions are an important tool in addressing human factors that are crucial to successfully managing the critically ill patient. Multidisciplinary paediatric simulation has been vital in identifying and addressing clinical governance issues such as above. Subsequently, these areas of improvement were escalated to the divisional management team and appropriate steps were taken to address them and minimise the risk to sick children.

\section{G315(P) DOCTORS, DEATH AND DIVERSITY: ARE WE AWARE OF THE CULTURAL NEEDS OF PARENTS DURING BEREAVEMENT CARE?}

M Williams, P Patel, E Perera, G Hann. Paediatric Department, North Middlesex University Hospital NHS Trust, London, UK

\subsection{6/archdischild-2015-308599.292}

Background When a child dies, the interactions between medical professionals and parents are remembered clearly and impact significantly both positively and negatively on the family's grieving process and eventual recovery. In our richly multi-cultural society it is an increasing challenge to provide sensitive, appropriate bereavement care.

Aims To determine whether paediatric doctors have basic knowledge of the cultural practices during end of life care associated with the five main religious affiliations present in our community (Muslim, Christian, Jewish, Hindu and No Religion). Methods An electronic questionnaire was sent to all paediatric trainees and consultants working in hospitals in one training sector of the UK. A combination of case based multiple choice questions and single answer questions were used. Results were collated anonymously.

Results 111 (28\%) of questionnaires were returned. Most doctors were able to identify Faith Leaders of Islam and Judaism (83\% and $81 \%$ respectively), and identify support for those of no specific religious affiliation, however only $5 \%$ of respondents could name a Faith Leader for Hinduism. Regarding actions at the time of death; Christian rituals were best understood (66\%) followed by Islamic (54\%), Hindu (44\%) and Jewish (30\%) traditions. Only $28 \%$ of respondents were aware that organ donation is not strictly forbidden in any of these religions. Overall, there was no relationship between training grade and cultural knowledge.

Conclusions As doctors we are not well informed about the predominant religious needs of our community. This is likely to impact adversely on our ability to offer appropriate bereavement care to parents. Our false beliefs about barriers to organ donation may also prevent us from offering this opportunity to newly bereaved parents. We plan to produce a nationally available training package in order to equip paediatric doctors with the knowledge to provide high quality, culturally sensitive end of life care.

\section{G316(P) CHRONIC AND COMPLEX CARE - A MODEL FOR GENERAL PAEDIATRIC LEADERSHIP IN A TERTIARY CENTRE}

JA Evans, R Greenslade, P Davis, G Body, J Van der Voort, D Tuthill, R Fish, S Struik, $\checkmark$ Rogers, C Norton, C Powell. Child Health, The Children's Hospital for Wales, Cardiff, UK

\subsection{6/archdischild-2015-308599.293}

Children with complex needs and their families coming into hospital with an acute admission may face a very unfamiliar environment despite perhaps having been under the care of community multidisciplinary teams for years. 